\title{
A QoS guaranteeing MAC layer protocol for the "underdog" traffic
}

\author{
Mahasweta Sarkar $^{1 *}$ and Christopher Paolini ${ }^{2}$
}

\begin{abstract}
With the tremendous boom in the wireless local area network arena, there has been a phenomenal spike in the web traffic which has been triggered by the growing popularity of real-time multimedia applications. Towards this end, the IEEE 802.11e medium access control (MAC) standard specifies a set of quality-of-service (QoS) enhancement features to ensure QoS for these delay sensitive multimedia applications. Most of these features are unfair and inefficient from the perspective of low priority (non-real time) traffic flows as they tend to starve the non-real time flows depriving them of appropriate channel access, hence throughput. To that extent, this article proposes a MAC protocol that ensures fairness in the overall network performance by still providing QoS for realtime traffic without starving the "underdog" or non-real-time flows. The article first presents analytical expressions supported by Matlab simulation results which highlight the performance drawbacks of biased protocols such as 802.11e. It then evaluates the efficiency of the proposed "fair MAC protocol" through extensive simulations conducted on the QualNet simulation platform. The simulation results validate the fairness aspect of the proposed scheme.
\end{abstract}

\section{Introduction}

Financial organizations, business houses and healthcare facilities have recently and repeatedly complained against network resource hogging by multimedia traffic when a minority section of their staff chooses to stream a video clip on Youtube which sabotages the transmission of an important data file like a patient's health record or a crucial email exchange [1,2]. This article investigates into alleviating this situation. With the widespread deployment of wireless local area networks (WLAN) in diverse environments, the demand for supporting a diverse range of applications is becoming increasingly important. Performance sensitive traffic such as voice and video applications require stringent delay constraints while data packets of a file transfer application, for example, can operate over a much broader delay and throughput requirement. To provide differentiated service to several such different categories of traffic, the IEEE 802.11e medium access control (MAC) standard [3] has the provision of traffic classification and prioritization. The standard classifies network

\footnotetext{
* Correspondence: msarkar2@mail.sdsu.edu

'Electrical and Computer Engineering, San Diego State University, 5500

Campanile Drive, San Diego, CA 92182, USA

Full list of author information is available at the end of the article
}

traffic into four different priority level or access categories (ACs). Each QoS-enabled station has four ACs, two high priority (HP) queues and two low priority (LP) queues. The packets delivered from the higher layers are tagged with priority values and en-queued into the corresponding priority queue according to the mapping illustrated in Table 1.

Each $\mathrm{AC}$ has its own transmit queue and its own set of AC parameters. Figure 1 shows a model where nodes maintain separate queues for each $\mathrm{AC}$ and packets at the head-of-line (HOL) of each queue contend for channel access using AC-specific parameters [4] which are more favorable to HP traffic than the LP traffic. The hybrid coordinator function (HCF)-controlled channel access (HCCA) mechanism is defined for parameterized QoS support. It uses a QoS-aware centralized coordinator, called the hybrid coordinator $(\mathrm{HC})$ allocated with the QoS-enabled access point of the QoS-enabled basic service set (BST) and has highest priority to access the wireless medium to issue polls to stations to provide limited-duration-controlled access phase for contentionfree transmission of QoS data. The HCF operates during the CP and CFP durations for providing QoS support for strict real-time applications. 
Table 1 User priority to access category mapping

\begin{tabular}{ccc}
\hline User priorities & ACs & Designation \\
\hline 1 & AC_BK & Background \\
2 & AC_BK & Background \\
0 & AC_BE & Best effort \\
3 & AC_VI & Video \\
4 & AC_VI & Video \\
5 & AC_VI & Video \\
6 & AC_VO & Video \\
7 & AC_VO & Video \\
\hline
\end{tabular}

Such a mechanism facilitates differentiated QoS where $\mathrm{HP}$, performance intensive traffic such as voice and video applications will enjoy less delay and greater throughput, compared to LP traffic (e.g., file transfer) $[5,6]$. The QoS features in IEEE 802.11e raise two related concerns. First, these mechanisms can often be unfair and inefficient from the perspective of nodes carrying LP traffic. Second, selfish nodes can gain enhanced performance by classifying LP traffic as HP, potentially destroying the QoS capability of the system.

We envision a system where majority of traffic is nonreal time, for example, in organizations like the healthcare industry, stock markets, and educational institutions, the bulk of the traffic still comprises of non-realtime flows. In these scenarios, it becomes essential to provide acceptable performance metrics for these nonreal-time traffic in the face of growing real-time multimedia traffic. The 802.11 e MAC scheme could have been justified if the majority of traffic in the system was real time. However, in these scenarios where the major chunk of network traffic is non-real time, the protocol will starve the non-real-time traffic which is the dominant traffic in most of these organizations and can present critical performance issues and diminish user satisfaction if not handled smartly [1,2]. Even a lone real-time flow can hog the network and starve the nonreal-time flows thereby drastically affecting the network performance [7]. This article raises the following concerns: (i) will the standard still favor HP traffic at the cost of LP traffic starvation, especially when the network traffic is LP-centric? (ii) what will happen if the applications start falsely classifying their traffic as HP in pursuit of preferential service [8]? Such instances might destroy the QoS capabilities of the network. The research community has raised concern over these issues of fairness [8-11]. The standard does not address these issues as it mainly deals with HP traffic, for which it allocates a major share of its resources.

This motivates us to propose a MAC protocol that does not starve the LP traffic or "underdog" traffic in face of HP traffic. Our scheme especially prevents resource hogging by the few HP traffic flows even when the predominant traffic in the network is LP. In this article, we thereby propose a MAC scheme which imparts fairness to the traffic ("Underdog"), i.e., getting exploited at the cost of preferential service offered by the standard to real-time traffic. The purpose of designing this scheme is to prevent starvation of nonreal-time LP data traffic while still maintaining an acceptable quality-of-service (QoS) performance for real time, delay sensitive HP traffic. We do so by introducing a transmission opportunity for LP traffic in the contention-free phase (CFP) of an IEEE 802.11e MAC protocol. Traditionally, IEEE 802.11 e MAC would provision for only HP traffic transmission during the CFP. In our proposed MAC scheme, we advocate the introduction of transmission slots for LP traffic as well during CFP.

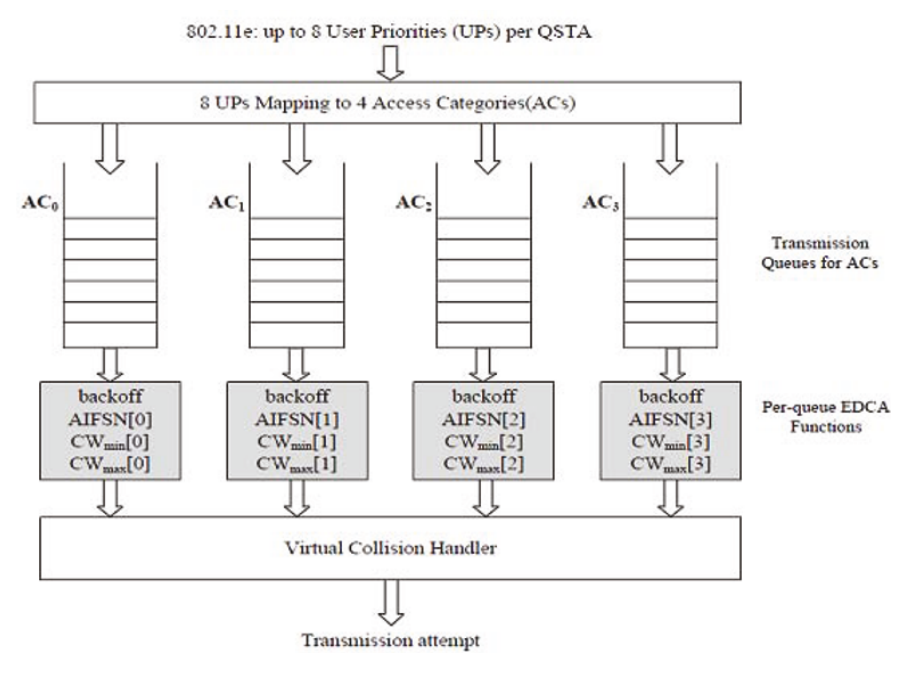

Figure 1 Access categories in 802.11e EDCA model. 
To explicitly understand the drawbacks of IEEE 802.11e (the standard which caters primarily to HP traffic) and thus motivate the need for a fair MAC protocol, we first analyze a hybrid-MAC scheme which mimics the 802.11e MAC in every essential respect. The analytical expressions attained for throughput and delay values of this hybrid MAC are discussed with the help of MATLAB simulation results. The drawbacks of an 802.11e-like MAC become apparent from these results. We thereby propose our fair MAC scheme. We perform extensive simulations on the network simulation platform QualNet to verify the feasibility and performance efficiency of our MAC scheme in comparison with the basic 802.11 e protocol. Simulation results validate the performance efficiency of our scheme.

The rest of the article is organized as follows. In Section 2, we provide a system model for our 802.11e-like Hybrid-MAC and derive throughput and delay expressions for the MAC along with MATLAB simulation results. In Section 3, we present and discuss our proposed MAC scheme. In Section 4, we present QualNet simulation results and provide an analysis and a comparative study of our scheme with 802.11e. We finally conclude the article in Section 5.

\section{Analysis of a hybrid-MAC}

We intend to derive analytical expressions for modeling throughput and delay characteristics of a MAC protocol that mimics the IEEE 802.11e in every essential respect. We do so by first proposing a simplified model of the IEEE 802.11e MAC.

\subsection{System model}

We set out to analyze the 802.11 e MAC protocol. We realize that an analysis of the exact scheme is cumbersome. We thus propose a hybrid-MAC model that resembles the 802.11e MAC in most essential respects. Our MAC model provides us with an abstraction of the essential features of 802.11e MAC, while avoiding the complex details of the latter. We believe that the insights obtained using our model are applicable to the 802.11e scenario. Our system model can be thought of as a hybrid MAC model which operates in both the contention and CFPs alternately, akin to a legacy 802.11 MAC protocol [4] with both its (a) distributed coordination function (DCF) and (b) point coordination function (PCF) modes enabled [4]. While DCF is based on the contention-based CSMA/CA mode of channel access, PCF is based on the polling mechanism. Limited QoS support in the legacy 802.11 standard is available through the use of the PCF. The DCF phase mimics the enhanced distributed channel access (EDCA) mechanism which is a contention-based channel access scheme while the PCF mimics the HCCA which is based on a polling mechanism. EDCA and HCCA are used to provide prioritized and parameterized QoS services, respectively, in 802.11e.

The network topology being modeled consists of a BSS of $N$ LP and $M$ HP traffic flows. We assume that each flow is generated by a node which we refer to as a STA (station), as done in the 802.11 standard. During the contention period (CP), each STA uses the basic access mechanism only, that is, no STA is assumed to be hidden from another STA and the RTS/CTS mechanism is not employed. During the contention-free period (CFP), the $M$ HP traffic STAs are placed in a circular queue and are polled sequentially by the PCF. The PCF implements two periods of channel access in a duration of time referred to as the "superframe": (i) a CFP and (ii) a CP. Figure 2 depicts an 802.11e superframe. The proportion of time allocated to each period within a superframe is not defined by the standard. The point coordinator subsystem residing in an AP continues to poll STAs in its polling list until the CFP duration expires.

\subsection{Modeling throughput}

Our analytical model for overall system throughput is a dimensionless multivariable function $S$ of $N, M, p$, and $\alpha$,

$$
S=S(N, M, p, \alpha)
$$

where $p$ is the probability of a successful frame transmission and $\alpha$ is a value between 0 and 1 that identifies the ratio of the time spent in the CFP to the total time spanned by a superframe which forms a repeating interval of contention and CFPs,

$$
\alpha=\frac{\mathrm{CFP}}{\mathrm{CFP}+\mathrm{CP}}
$$

As $\alpha$ tends toward 0, the BSS reverts to a contentiononly-based environment where the point coordinator is not used to poll STAs. With a non-zero $\alpha$, dimensionless throughput $S$ becomes a weighted sum of time spent in the CP and the CFP,

$$
S(N, M, p, \alpha)=(1-\alpha) S_{\mathrm{CP}}+\alpha S_{\mathrm{CFP}}
$$

We then apply the definitions of $S_{\mathrm{CP}}$ and $S_{\mathrm{CFP}}$ given in [12] for dimensionless throughput for each respective period,

$$
\begin{aligned}
& S_{\mathrm{CP}}=\frac{\bar{U}_{\mathrm{CP}}}{\bar{I}_{\mathrm{CP}}+\bar{B}_{\mathrm{CP}}} \\
& S_{\mathrm{CFP}}=\frac{\bar{U}_{\mathrm{CFP}}}{\bar{B}_{\mathrm{CFP}}}
\end{aligned}
$$




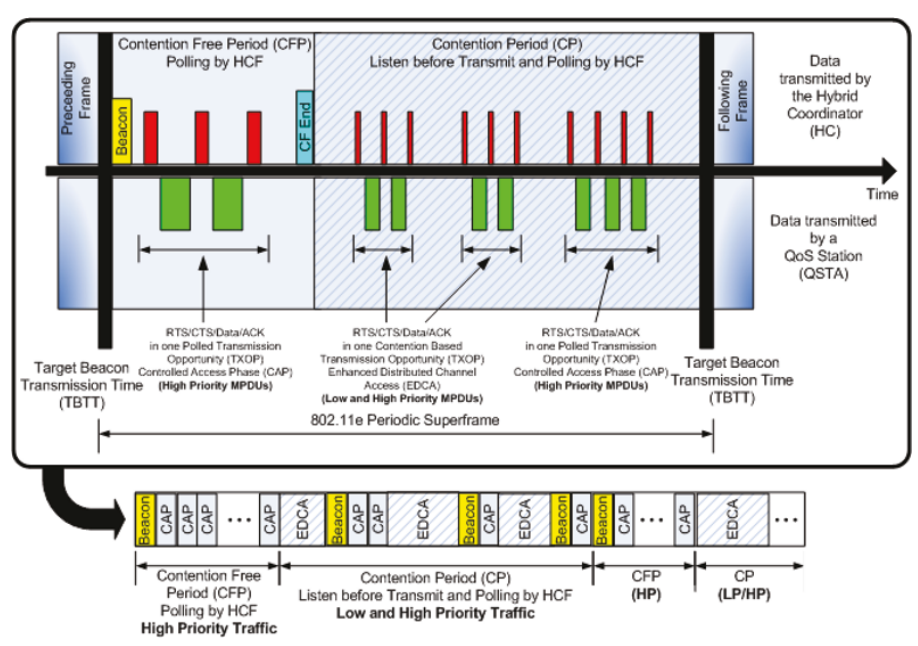

Figure 2 802.11e super frame showing HP traffic constrained to the CFP while LP and HP traffic compete for channel access during the CP. The HC in the CP also polls stations for HP traffic.

In Equation 4, $\bar{U}_{\mathrm{CP}}$ is the average duration of time the useful data are received by a STA during the CP, $\bar{I}_{\mathrm{CP}}$ is the average duration of time the channel remains idle during the $\mathrm{CP}$, and $\bar{B}_{\mathrm{CP}}$ is the average duration of time the channel is busy transmitting data, the overhead bits incurred by the data, and is handling collisions [12]. Equation 4 is then a dimensionless quantity between 0 and 1 that represents throughput efficiency as the ratio of time the channel is used for sending useful data to total time. We can extend this concept by defining $S_{\text {CFP }}$ in a similar way, with the exception that we exclude the idle term in the denominator since it is assumed that the channel is never idle during the CFP. The definitions of $\bar{U}_{\mathrm{CP}}, \bar{I}_{\mathrm{CP}}$, and $\bar{B}_{\mathrm{CP}}$ are extended from [12], with the modification that the total STA count has been replaced by $(N+M)$,

$$
\begin{aligned}
& \bar{U}_{\mathrm{CP}}=\frac{(N+M) T p}{(1-p)\left(1-(1-p)^{N+M}\right)} \\
& \bar{I}_{C P}=\frac{\sigma}{1-(1-p)^{N+M}} \\
& \bar{B}_{\mathrm{CP}}=\frac{T_{\mathrm{s}}}{(1-p)^{N+M}}
\end{aligned}
$$

In Equation 6, $T$ is the time spent in the $\mathrm{CP}$ transmitting useful data, that is, the ratio of the length in bits of packet payload $P$ (excluding the number of header and trailer bits, $H$ ) to the data rate $R$. The other time parameter, $T_{\mathrm{s}}$, in (8) is the time spent sensing the channel during a successful frame transmission. Substituting (6),
(7), and (8) into (4), we obtain, as in [12],

$$
S_{\mathrm{CP}}=\frac{(N+M) T p(1-p)^{N+M-1}}{T_{\mathrm{s}}+\left(\sigma+T_{\mathrm{s}}\right)(1-p)^{N+M}}
$$

The expression for $T_{\mathrm{s}}$ is given by

$$
T_{\mathrm{s}}=\mathrm{DIFS}+\frac{H+P}{R}+\mathrm{SIFS}+\frac{\mathrm{ACK}}{R}+2 \tau
$$

To derive Equation 10, we note that synchronized data exchange within the CFP are accomplished by polling STAs. The polling process is coordinated by the PCF implementation within an AP. When the CFP begins, the AP waits a brief duration of time known as a short interframe space (SIFS) which serves as a delay between beacon, data, acknowledgement, and end frames that are transmitted during the CFP. The value of SIFS varies by the particular 802.11 standard implemented by a transceiver. For $802.11 \mathrm{a}, \mathrm{b}$, and g, the values are 16, 10, and $10 \mu \mathrm{s}$, respectively. After waiting an initial SIFS, the AP commences with polling by transmitting a Data/CF-Poll frame to the first STA in a polling list. Data/CF-Poll frames serve a dual purpose by piggybacking data carried by the AP which, in an infrastructure mode network, is attached to a wired network via a wired Ethernet interface. The Data/ CF-Poll frame polls the receiving STA while simultaneously carrying higher layer datagrams originating from another STA within a BSS or a device external to a BSS via a wired LAN. The collision avoidance $(\mathrm{CA})$ mechanism of CSMA/CA cannot guarantee collisions will not occur. A collision can occur, for example, if two STAs compute exactly the same backoff time after detecting a channel idle for DCF interframe space duration (DIFS) and then transmit a MPDU when the backoff timer matures. To 
determine if a transmission resulted in a collision, each data frame (MPDU) must be acknowledged through the transmission of an ACK frame sent by the STA receiving a data frame. If a sending STA does not receive a corresponding ACK after waiting a SIFS period, the sending STA concludes a collision occurred and will repeat the transmission. DIFS values for 802.11a, b, and g are 34, 50, and either 28 or $50 \mu \mathrm{s}$, depending on slot time, respectively. In IEEE $802.11 \mathrm{~g}$, the slot time can be either $9 \mu$ if no legacy $802.11 \mathrm{~b}$ STAs are present in the BSS, or $20 \mu \mathrm{s}$ if the BSS has a mix of $802.11 \mathrm{~b}$ and $802.11 \mathrm{~g}$ STAs. DIFS is a function of SIFS and is computed according to

$$
\text { DIFS }=\text { SIFS }+2 \sigma
$$

where $\sigma$ is the slot time defined to be twice the maximum propagation time $\tau$. The slot time is therefore an amount of time a STA requires to determine if another STA has accessed the channel at the start of the previous slot. Slot time values for 802.11a and b are 9 and $20 \mu \mathrm{s}$, respectively, for a PHY that uses a direct sequence spread spectrum (DSSS) modulation technique and $50 \mu$ s for a PHY that uses a frequency hopping spread spectrum (FHSS) transmission method. Acknowledgement frames may also piggyback data originating from a receiving STA and intended for another STA in the BSS or an external device. If the point coordinator fails to receive a response from a polled STA within a PCF interframe space (PIFS) period of time, the PCF will move on and poll the next STA in its polling list. PIFS is also function of SIFS and is computed according to

$$
\text { PIFS }=\text { SIFS }+\sigma
$$

and thus the values for $802.11 \mathrm{a}, \mathrm{b}$, and $\mathrm{g}$ are 25,30 , and either 19 or $30 \mu \mathrm{s}$, respectively. The PIFS duration also serves as a gap between the CP and CFP. From (11) and (12) we have the following inequality

$$
\text { SIFS }<\text { PIFS }<\text { DIFS }
$$

which prevents the PCF from transmitting a poll frame in between a Data/CF-Poll and Data/CF-ACK transaction.

Given the definitions of SIFS and DIFS, Equation 10 can be understood as the sum of times required to conduct a successful packet transmission in the CP: the STA must first wait a DIFS amount of time to detecting a channel idle before proceeding to transmit, then an $(H$ $+P) / R$ amount of time to for an interface to transmit a packet consisting of $H$ header and trailer bits and $P$ payload bits at a data rate $R$, then a $\tau$ amount of time for propagation of the data packet, then a SIFS amount of time before the receiving STA's interface can transmit an acknowledgement frame, then $(\mathrm{ACK} / R)$ time to transmit the acknowledgement frame, and finally another $\tau$ amount of time for propagation of the acknowledgement.

Our derivation of $S_{\mathrm{CFP}}$ proceeds in a similar way to that of $S_{\mathrm{CP}}$. Let $q$ represent the probability a STA has a non-null data frame to transmit during the CFP. $\bar{U}_{\mathrm{CFP}}$ is the average time spent during the CFP to transmit useful data. By useful data we mean data bits and not bits belonging to beacon, pure ACK, and CF-End frames. If we denote $P_{\mathrm{CFP}}$ as the number of data bits transmitted during the CFP, then

$$
\bar{U}_{\mathrm{CFP}}=\frac{P_{\mathrm{CFP}}}{R}
$$

where $R$ is the fixed transceiver data rate.

To derive an expression for the mean time the channel is busy in the CFP during a successful polling transaction, denoted $\bar{B}_{\mathrm{CFP}}$, we need to account for all the individual frame transmissions namely, $\mathrm{CF}_{\mathrm{Beacon}}, \mathrm{CF}_{\mathrm{Poll}}, \mathrm{CF}_{\mathrm{ACK}}$, and $\mathrm{CF}_{\text {Null }}$ which represent the lengths of the beacon, Data/ CF-Poll, Data/CF-ACK, and CF-NULL frames, respectively. CF-Null frames are transmitted by a polled STA if the STA does not have any pending data to send, $\tau$ is the propagation delay of the wireless LAN, and $H$ is the length of the header and frame check sequence (FCS) of an 802.11 frame. The first term in Equation 15 is the time required for the hybrid coordinator $(\mathrm{HC})$ operating in an access point to transmit a beacon frame and for the beacon to propagate. The second term in (15) is the time required to poll all the LP and HP stations being coordinated by the HC during the CFP. The third term is the probability all the stations have a non-null data frame waiting to transmit upon being polled. The summation in parenthesis is the time required for the corresponding station to acknowledge the poll by returning a combined Data/CF-ACK frame. The fourth term then accounts for the time required for all the stations that do not have data to send and will transmit a CF-NULL frame back to the $\mathrm{HC}$ upon being polled.

$$
\begin{aligned}
\bar{B}_{\mathrm{CFP}} & \left(\mathrm{PIFS}+\frac{\mathrm{CF}_{\text {Beacon }}}{R}+\tau\right)+ \\
& (N+M)\left(\mathrm{SIFS}+\frac{H+P+\mathrm{CF}_{\mathrm{Poll}}}{R}+\tau\right)+ \\
& (N+M) q^{(N+M)}\left(\mathrm{SIFS}+\frac{H+P+\mathrm{CF}_{\text {Data } / \mathrm{ACK}}}{R}+\tau\right)+ \\
& (N+M)(1-q)^{(N+M)}\left(\mathrm{SIFS}+\frac{H+P+\mathrm{CF}_{\text {Null }}}{R}+\tau\right)+ \\
& \left(\mathrm{SIFS}+\frac{\mathrm{CF}_{\text {End }}}{R}+\tau\right)
\end{aligned}
$$

\subsection{Modeling delay}

Our analytical model for overall system delay is a dimensionless multivariable function $D$ of $N, M, p$, and $\alpha$, 


$$
D=D(N, M, p, \alpha)
$$

Observe that

$$
0<\frac{D_{\text {ideal }}}{D_{\text {actual }}} \leq 1
$$

where $D_{\text {ideal }}$ is the theoretical minimum delay a STA can experience in a superframe while $D_{\text {actual }}$ is the true delay experienced. If we define $D$ such that

$$
D=\left(1-\frac{D_{\text {ideal }}}{D_{\text {actual }}}\right)
$$

Then $D \rightarrow 0$ as the actual delay approaches the ideal and $D \rightarrow 1$ as actual delay diverges from the ideal. We first consider delay incurred by the DCF. Ideal delay in the $\mathrm{CP}$ can be expressed as the sum of ideal HOL delay and ideal queuing delay,

$$
D_{\text {ideal }}=D_{\text {ideal }}^{\text {HOL }}+D_{\text {ideal }}^{\text {Queuing }}
$$

where $D_{\text {ideal }}^{\mathrm{HOL}}$ represents the minimum time required in the CP to transmit an 802.11 frame successfully, upon the first attempt, and is equal to $T_{\mathrm{s}}$. Ideal queuing delay is given by the Pollaczek-Khinchine formula [12]

$$
D_{\text {ideal }}^{\text {Queuing }}=\frac{\rho}{2 \mu(1-\rho)}\left(1+c v^{2}\right)
$$

that describes the mean time a frame waits in queue to be serviced by the MAC, where the queue is modeled as a M/G/1 queue (a single server with frame arrivals having a Poisson distribution and service time having a general distribution). Total actual delay $D_{\text {actual }}$ is modeled as the sum of (20) and an expression for the expected value of HOL delay which takes into account backoff delay.

In Equation 21, $\beta$ is the average physical time between two decrements of the backoff counter, $\mathrm{CW}_{\min }$ is the minimum contention window size, $P_{s}=(1-p)^{M+N-1}$ is the probability a STA's frame transmission is successful, and $r_{\max }$ is the maximum number of retransmissions permitted. In our simulation, $\mathrm{CW}_{\min }$ is set to $2^{4}$ and $\mathrm{CW}_{\max }$ is set to $2^{10}$ which are the values used by a PHY that employs a FHSS method of transmitting radio signals. Considering now the PCF, each STA has an opportunity to transmit when polled while the CFP is in progress. If the maximum predetermined duration of the CFP in a given superframe expires before every STA has been polled, STAs that were not given an opportunity are more likely to be polled in the following CFP as the PC uses a circular queue to schedule station polling.

$$
\begin{aligned}
& E\left[D_{\text {actual }}^{\text {HOL }}\right]= T_{s}+\beta\left[\frac{\mathrm{CW}_{\min }}{2\left(1-\left(1-P_{s}\right)^{r_{\max }+1}\right)}\right] \\
& {\left[\frac{P_{s}\left(1-\left(2\left(1-P_{s}\right)\right)^{r_{\max }+1}\right)}{1-2\left(1-P_{s}\right)}-1-\left(1-P_{s}\right)^{r_{\max }+1}\right]+} \\
& T_{\mathrm{s}}\left[\frac{1-P_{s}}{P_{s}}\right]\left[\frac{\left(1-P_{s}\right)^{r_{\max }}\left(-P_{s} r_{\max }-1\right)+1}{1-\left(1-P_{s}\right)^{r_{\max }+1}}\right]
\end{aligned}
$$

Also, $r_{\max }$ is defined as

$$
r_{\max }=\log _{2}\left(\mathrm{CW}_{\max } / \mathrm{CW}_{\min }\right)
$$

since the number of different contention window sizes will be the exponent of the ratio of $\mathrm{CW}_{\max }$ to $\mathrm{CW}_{\min }$. Equation (22) therefore gives the maximum number of retransmission attempts that will be made, if the initial transmission should result in a collision. For a FHSS based PHY, $r_{\max }$ is 6 .

$D_{\text {ideal }}^{\mathrm{HOL}}$ in (21) is without any backoff delay,

$$
D_{\text {ideal }}^{\mathrm{HOL}}=T_{\mathrm{s}}
$$

Let $\psi$ be a random variable and $E[\psi]$ represent the expected value (a number in the range $[0,2312]$ ) of the size of the body of data within an 802.11 frame transmitted by a polled station during the CFP, then

$$
\Psi=34+E[\psi]
$$

since 34 equals the maximum number of bits that comprise an 802.11 MAC header with the cyclic redundancy check (CRC) (A.K.A FCS) field included (see Figure 3).

Assuming the length of data in frames transmitted during the CFP follows a discrete uniform distribution (i.e., all frame lengths within the range $[0,2312]$ are equally likely), $\bar{\Psi}=E[\Psi]=34+(0+2312) / 2=1190$ bits and the mean total time for one CFP is given by

$$
\begin{aligned}
\bar{T}_{\mathrm{CFP}}= & \text { PIFS }+\frac{\mathrm{CF}_{\text {Beacon }}}{R}+(N+M) \frac{\left(\bar{\Psi}_{\mathrm{PC}}+\bar{\Psi}_{\mathrm{STA}}\right)}{R} \\
+ & {[2(N+M)+1] \mathrm{SIFS}+\frac{\mathrm{CF}_{\text {End }}}{R}+2[N+M+1] \tau } \\
\bar{T}_{\mathrm{CFP}} & =\text { PIFS }+\frac{\mathrm{CF}_{\text {Beacon }}}{R}+(N+M) \frac{\left(\bar{\Psi}_{\mathrm{PC}}+\bar{\Psi}_{\mathrm{STA}}\right)}{R} \\
+ & {[2(N+M)+1] \mathrm{SIFS}+\frac{\mathrm{CF}_{\text {End }}}{R}+2[N+M+1] \tau }
\end{aligned}
$$

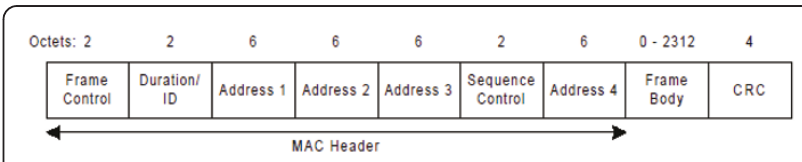

Figure 3 Format of an 802.11 MAC frame. 
In Equation 25, we account for polling frames that may either be CF-Poll with no data (subtype 6 or 0110 ) or CFPoll + Data (subtype 2 or 0010$)$ as $(N+M) \bar{\Psi}_{\text {PC }}$ represents the mean length of polling frame bits transmitted by the point coordinator during the CFP. Similarly, we account for acknowledgement frames that may be CFACK with no data (subtype 5 or 0101) or CF-ACK + Data (subtype 1 or 0001$)$ as $(N+M) \bar{\Psi}_{\text {STA }}$ represents the mean length of acknowledgement frame bits transmitted by all the stations during the CFP. The remaining terms in (25) follow from (15) and account for interframe delays, management and control frames, and propagation times.

Let $D_{\text {CFP }}$ represent the average time a frame must wait at the HOL once the CFP begins. The first polled station must wait

$$
\mathrm{PIFS}+\frac{\mathrm{CF}_{\text {Beacon }}}{R}+2(\mathrm{SIFS}+\tau)+\frac{\Psi_{\mathrm{PC}}}{R}
$$

time duration before transmitting a frame. The second station must wait the time given in (26) plus

$$
2(\mathrm{SIFS}+\tau)+\frac{\Psi_{\mathrm{STA}}+\Psi_{\mathrm{PC}}}{R}
$$

amount of time before transmitting a frame. Thus, from (26) and (27), the average time a station must wait before transmitting a frame is

$$
\begin{aligned}
\bar{D}_{\mathrm{CFP}} & =\mathrm{PIFS}+(N+M)(\mathrm{SIFS}+\tau)+\frac{\mathrm{CF}_{\text {Beacon }}}{R} \\
& +\frac{1}{R}\left[\left(\frac{N+M}{2}\right) \Psi_{\mathrm{PC}}+\left(\frac{N+M}{2}-1\right) \Psi_{\mathrm{STA}}\right]
\end{aligned}
$$

From (19), (20), (23), and (28) we now have

$$
\bar{D}_{\text {ideal }}=T_{\mathrm{s}}+\frac{\rho}{2 \mu(1-\rho)}\left(1+c v^{2}\right)+\bar{D}_{\mathrm{CFP}}
$$

Accounting for backoff delay, the actual delay is modified to give $D_{\text {actual }}$ which is shown in (25).

$$
\begin{aligned}
& \bar{D}_{\text {actual }}=T_{\mathrm{S}}+\beta\left[\frac{\mathrm{CW}_{\min }}{2\left(1-\left(1-P_{\mathrm{s}}\right)^{r_{\max }+1}\right)}\right] \\
& {\left[\frac{P_{s}\left(1-\left(2\left(1-P_{s}\right)\right)^{r_{\max }+1}\right)}{1-2\left(1-P_{s}\right)}-1-\left(1-P_{s}\right)^{r_{\max }+1}\right]} \\
& +T_{s}\left[\frac{1-P_{s}}{P_{s}}\right]\left[\frac{\left(1-P_{s}\right)^{r_{\max }}\left(-P_{s} r_{\max }-1\right)+1}{1-\left(1-P_{s}\right)^{r_{\max }+1}}\right] \\
& +\frac{\rho}{2 \mu(1-\rho)}\left(1+c v^{2}\right) \\
& + \text { PIFS }+(N+M)(\operatorname{SIFS}+\tau)+\frac{C_{\text {Beacon }}}{R} \\
& +\frac{1}{R}\left[\begin{array}{c}
\mathrm{CF}_{\text {Beacon }}+\left(\frac{N+M}{2}\right) \Psi_{\mathrm{PC}} \\
+\left(\frac{N+M}{2}-1\right) \Psi_{\mathrm{STA}}
\end{array}\right]
\end{aligned}
$$

\subsection{Analysis of the hybrid-protocol simulation results}

We evaluated the accuracy of our analytical expressions for dimensionless throughput and normalized delay by developing a MATLAB simulation based on our derivations. Figures 3 and 4 represent the dimensionless throughput and normalized delay values as the number of HP STAs in the BSS increases with varying superframe period duration $\alpha$. We see that the value of $\alpha$ has a significant effect on system performance with respect to throughput and delay. Similarly, the collision probability impacts throughput and delay. Figures 3 and 4 show a surface plot that quantifies the relationship between collision probability, number of HP users, and the effect these parameters have on system delay and throughput, respectively. When the system operates in equal duration of CP and CFP (i.e., $\alpha=0.5$ ), the throughput decreases with an increase in the number of HP users, gradually approaching an asymptote. This can be explained by the fact that an increasing number of HP users create higher contention in the CP phase leading to longer backoff time and thereby a drop in throughput and an increase in delay, as seen in Figure 4. Interestingly, the delay value also approaches an asymptote as the number of HP users in the BSS increase (when $\alpha=0.5$ ). In Figure 3, we see that, as the number of HP stations increases, a saturation condition at normalized delay $D=1$ is attained with lower values of collision probability $p$.

With respect to Figures 4 and 5, collision probability $p$ is defined as the probability a given frame transmission attempt is unsuccessful due to a collision occurring in the CP. Looking at Figure 3, one can see that for a small number of HP stations, the directional derivative $d D / d p$ is much less than it is for a large number of HP stations. Because the rate of change in delay increases faster with respect to station count as collision probability increases, a saturation condition will arise sooner in a BSS with many high priority traffic stations if stations begin to experience a greater number of collisions in the contention period. Similarly, in Figure 4, we see how small changes in collision probability can greatly affect throughput as the HP station count increases. We also see the appearance of an optimal throughput contour along the maxima of the surface $S$.

\section{The proposed fair MAC scheme}

Providing fair channel access opportunities to both HP and LP traffic such that adequate throughput is enjoyed by non-real time (or LP) flows while still supporting the QoS constraints of real-time traffic (or HP flows) is the main objective of this study, especially under scenarios where the bulk of network traffic is non-real time. Thus, we have designed a scheme that would be suitable for networks dominated by LP traffic and one that would 


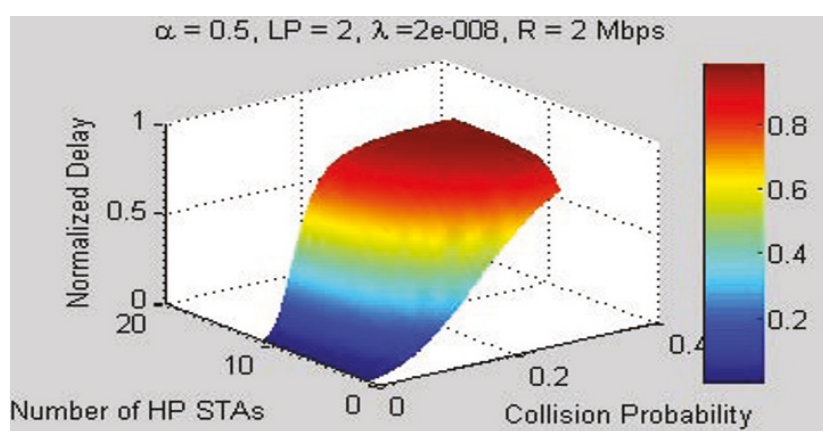

Figure 4 Normalized delay surface plot $D=D(\mathrm{HP}, p)$

eventually revert back to normal 802.11e functionality in the absence of LP traffic. Before we delve into the details of our fair MAC scheme, it is worthwhile to examine the existing IEEE 802.11e MAC protocol.

\subsection{Examining IEEE 802.11e MAC protocol}

To enhance the QoS support, IEEE 802.11e introduces a protocol called the HCF which includes two medium access mechanisms: contention-based channel access and controlled channel access which are referred to as the EDCA and HCCA. With 802.11e, there are two phases of operation within a superframe, i.e., the CP and a CFP. Each superframe begins with a control frame called the Beacon frame followed by the $\mathrm{CP}$ and then the CFP. Figure 2 pictorially depicts a typical 802.11e superframe.

The EDCA is used in the CP only, while the HCCA is used in both phases. QoS polling for HCCA can take place during $\mathrm{CP}$ as well. EDCF and HCCA together support up to eight priority traffic classes (TC). Each TC starts with a backoff after detecting the channel being idle for an arbitration interframe space (AIFS) period of time. The AIFS can be chosen individually for each TC and thus provides a deterministic priority mechanism between the TCs. Thus, a transmit opportunity (TXOP) almost always is given to the TC with the highest priority. During the $\mathrm{CP}$, access is governed by EDCF, though the hybrid coordinator ( $\mathrm{HC}$-generally co-located within the AP) can initiate HCF access at any time. During the CFP, the $\mathrm{HC}$ issues a QoS CF-Poll frame to a particular station to give it a TXOP, specifying the start time and maximum duration. No station attempts to gain access to the medium at this time and thus the station to which the CFP-poll frame was sent has unhindered access to the medium. The HC has available, over time, a snapshot view of the per-TC, per station, queue length information in the cell, including that of the AP itself. This information is sent to the HC by stations periodically. With this information, the $\mathrm{HC}$ decides which station (including itself) to allocate TXOPs during the CFP. At minimum, the following needs to be considered: (a) priority of the TC, (b) required QoS for the TC (low jitter, high bandwidth, low latency, etc.), (c) queue lengths per TC, (d) queue lengths per station, (e) duration of TXOP available and to be allocated, and (f) past QoS seen by the TC. Thus, even during the HCCA (as during the EDCA), TXOPs are given to traffic of HP as well.

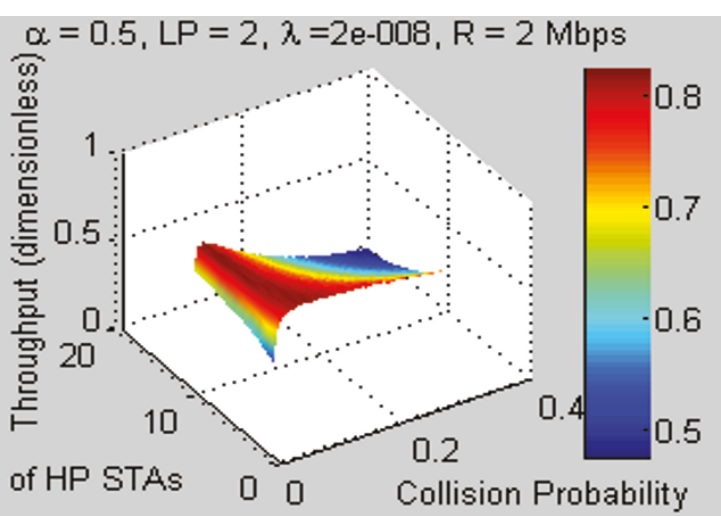

Figure 5 Dimensionless throughput surface plot $S=S(\mathrm{HP}, p)$. 


\subsection{Motivating the need for a FAIR MAC scheme}

Performance analysis of the QoS enhancements of 802.11e has been demonstrated in [13-15]. Simulation studies in $[16,17]$ show that the EDCA provides significant improvements for HP traffic; however, these improvements are typically provided at the cost of worse performance for LP traffic. This is precisely the problem that we identify and help mitigate in this article. We argue that a protocol as biased as $802.11 \mathrm{e}$ (toward HP traffic) can be detrimental to system performance, especially when the traffic classification (as to who is HP traffic and who is LP) is left to applications. Any rational, self-serving LP application will realize that the system "does not care" about LP traffic and might want to falsely classify its traffic as HP traffic in pursuit of better performance. This would potentially breakdown the entire paradigm of delivering QoS to the ones who need it the most. Thus, we recommend in this article that TXOPs be given to both HP and LP traffics-not equally (that would not be fair to the HP traffic) but at least partially, such that LP traffic is not robbed completely of transmission opportunities in the presence of HP traffic. We take an extremely unconventional approach and propose that we use the CFP of a superframe to be dedicated to transmission of LP traffic along with HP traffic by means of polling LP users by the HC. Obviously the TXOP duration should not be too long so as to increase the delay encountered by the HP users beyond what is acceptable. The CP phase remains a solely contention phase where HP traffic gets preferential channel access over LP traffic. Figure 6 denotes our recommended scheme.

\subsection{Our FAIR MAC scheme}

Conventionally, contention-based channel access schemes have been used for LP data transmission whereas "polling" and thereby dedicated channel access schemes have been thought of as the most appropriate way of transmitting HP (delay-sensitive) data. It is a well-established fact that if a MAC layer protocol has to cater to various types of traffic (both HP and LP), it is imperative that it employs both contention-based and polling channel access mechanisms. Thus, our fair MAC scheme alternates between a contention-based channel access mechanism, which we refer to as the $\mathrm{CP}$, and a polling-based channel access scheme, which we refer to as the CFP as shown in Figure 6. Our system offers channel access opportunities to both traffic types (HP and LP) during the contention period, allocating higher preference to the HP traffic to grab the channel over the LP traffic. However, deviating from the norm, during the CFP, LP traffic is included in the polling list and thus polled by the $\mathrm{HC}$ along with the HP traffic. The duration of the CFP is equally distributed to allocate transmission time for all traffic flows in the network. The polling scheme is implemented in a circular queue such that all traffic flows gets polled almost equally. The $\mathrm{HC}$, co-located with the AP, polls every station in the polling list starting with the traffic flow which has the highest priority and subsequently servicing the traffic flows on the polling list in the descending priority order till the lowest priority traffic flow is served. The HP flows still retain their precedence in the queue over the LP flows. However, such dedicated service during the CFP incentivizes LP traffic to deter from falsely classifying itself as HP and thus preserves system sanctity.

During the CP, a node with packets to transmit contends for channel access with a certain probability. QoS differentiation is enforced by allowing packets in HP queues to contend for channel access with higher probability than packets in LP queues. We assume that nodes are transmitting to an AP that can invoke a CFP by issuing a poll request to one or more nodes. These polled nodes can then transmit without any contention. Users can classify their applications as either HP or LP. Users are expected to take advantage of the MAC's QoS features by declaring their delay sensitive applications as HP, and delay tolerant applications as LP. The AP needs to decide what fraction of time the system will spend in the contention and CFPs. Our protocol is very similar to

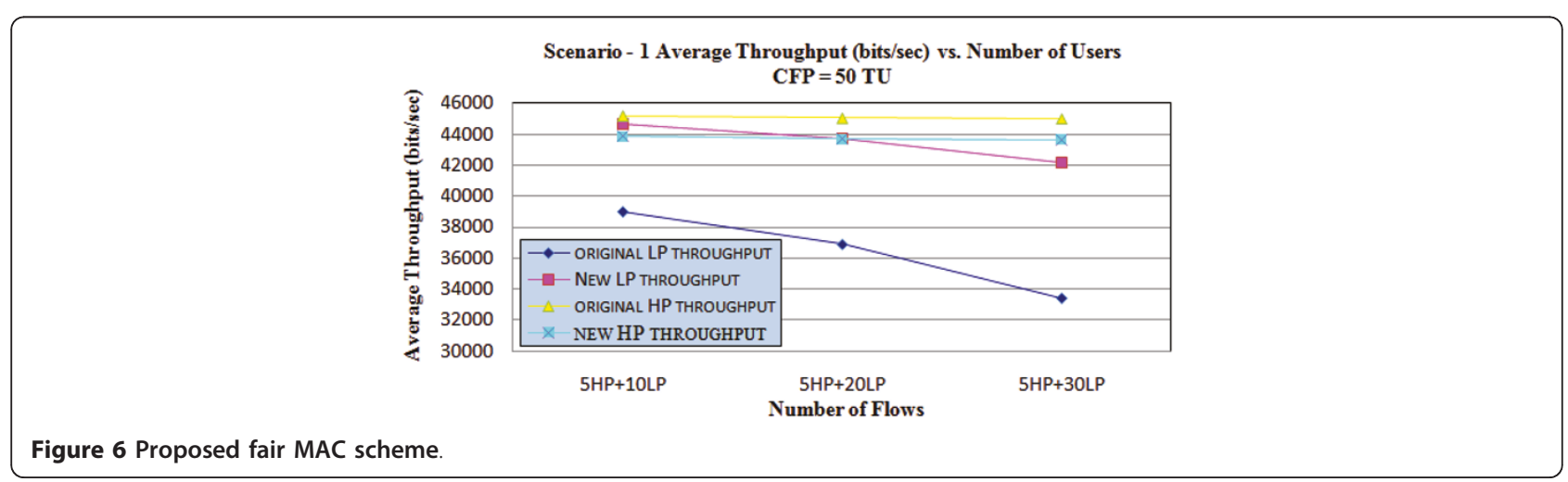


802.11e's HCF, with the CP corresponding to 802.11e's random access or EDCA functionality and the CFP corresponding to $802.11 \mathrm{e}$ 's polled access or HCCA functionality [18]. More specifically, our system corresponds to the HCCA/EDCA mixed mode [3] of operation.

The vast majority of moderate-rate delay sensitive HP applications (such as VoIP and moderate resolution video streaming) and delay tolerant LP applications (e.g., file transfer and email) can be supported by the random access or contention functionality of 802.11 e. If the number of users with delay sensitive traffic is relatively large, then polling or contention-free access is inappropriate because of the large delay incurred in waiting for one's turn [17]. Therefore, from the HP user's viewpoint, it is more advantageous to operate in the $\mathrm{CP}$ rather than the CFP. On the other hand, operating the network mainly in a CP is both unfair and inefficient as far as LP applications are concerned. It is unfair because HP applications will obtain better throughput than LP applications as they contend for channel access more aggressively. It is inefficient because, even in the absence of HP applications, LP applications are forced to be conservative in accessing the channel. Thus, arises the interesting dilemma of how long should these CP and CFP periods be chosen such that system performance is maximized. We choose to investigate this problem in our future study.

It is also noted that polling is known to be very efficient throughput-wise, but leads to large delays because a user has to wait for his/her turn to transmit [17]. Since LP traffic is delay-tolerant, polling is an efficient method to serve such traffic. Another consequence of the throughput efficiency of polling is that the system does not need to spend too much time in the CFP to serve LP users. Thus, the negative impact of our scheme on HP users is mild. Most of the time the system is in the CP where HP users can enjoy good delay performance of prioritized random access. Our incentive mechanism exploits the difference in performance required by HP and LP applications, to simultaneously satisfy QoS requirements for all users. HP applications, such as VoIP, have tight delay constraints but do not require very high throughput. LP applications such as file transfer have no particular delay constraints but require relatively high throughput for reasonable session completion times. Polling LP users during the CFP ensures that these users are guaranteed a certain minimum level of throughput, ensuring there is no motivation for LP users to falsely declare their traffic type as HP. This in turn implies that HP users encounter decreased interference from LP users during the CP leading to better delay performance.

It is to be noted that the duration of the CFP phase has a significant impact on the delay encountered by the
HP traffic. This is because, the longer the CFP (to accommodate the several LP flows in a network), the more the time required for the system to transition into the $\mathrm{CP}$, thereby making the HP traffic wait for a longer period of time to get an opportunity to transmit their delay sensitive data. We intend to address this issue in a quantitative manner in our future study. In Section 4, we provide a numerical analysis of the above fact. We want to emphasize that an absence of LP traffic flow in the network will make our scheme behave exactly in the standard 802.11e fashion. Thus, no undue delay will be encountered by the HP traffic flows. In summary, the extra opportunity to transmit data by the LP flows during the CFP phase leads to significant increase in their throughput with minor dent in the delay performance of the HP flows.

\section{Simulation results}

We evaluated our proposed fair MAC scheme using the network simulation platform QualNet 4.5 [18]. Our network topology was comprised of several wireless stations (or nodes) and one AP, all located within each others' "hearing" range (i.e., every station is able to detect a transmission from any other station in the network). The nodes were placed in the default terrain with default dimension settings. Each simulation has been run for $600 \mathrm{~s}$ and each reported value has been averaged over 15 runs. The simulation results were analyzed using the QualNet analyzer.

Table 2 enumerates the simulation parameters that we used. It is worth mentioning that some of the system parameters in a real network-like contention window duration-are a function of the physical (PHY) layer protocol. We present some realistic values of such system parameters in Table 3[19]. We were mainly interested in analyzing the throughput and end-to-end delay characteristics of our protocol in comparison to the IEEE 802.11e standard MAC protocol. We created two distinct network scenarios-network scenario I was comprised of a fixed number of HP traffic flows (5) with an increasing number of LP traffic flows. Specifically, we

\section{Table 2 Simulation parameters}

\begin{tabular}{ll}
\hline MAC protocol & 802.11e with HCCA enabled \\
\hline $\begin{array}{l}\text { PHY/radio } \\
\text { model }\end{array}$ & $802.11 \mathrm{~b}$-data rate $2 \mathrm{Mbps}$ \\
\hline Beacon interval & 200 time units (TU) \\
\hline CFP duration & $50 \mathrm{TU}, 160 \mathrm{TU}$ \\
\hline $\begin{array}{l}\text { Simulation } \\
\text { duration }\end{array}$ & $600 \mathrm{~s}$ \\
\hline Seed & $1-15$ \\
\hline $\begin{array}{l}\text { Type of traffic } \\
\text { source }\end{array}$ & CBR with precedence 5, 6, 7 for HP traffic CBR with \\
\hline
\end{tabular}


Table 3 System parameter values for three different PHYs as specified by IEEE 802.11 standard [19]

\begin{tabular}{llll}
\hline PHY & Slot time $(\boldsymbol{\mu s})$ & CW $_{\min }$ & CW $_{\max }$ \\
\hline FHSS & 50 & 16 & 1024 \\
\hline DSSS & 20 & 32 & 1024 \\
\hline R & $8 \mu$ & 64 & 1024 \\
\hline
\end{tabular}

studied three distinct network configurations, namely (a) $5 \mathrm{HP}$ and $10 \mathrm{LP}$ flows, (b) $5 \mathrm{HP}$ and 20 LP flows, and (c) $5 \mathrm{HP}$ and 30 LP flows. Network scenario II was comprised of a fixed number of LP traffic flows (20) with an increasing number of HP traffic flows. Specifically, we studied three distinct network configurations, namely (a) $10 \mathrm{HP}$ and $20 \mathrm{LP}$ flows, (b) $15 \mathrm{HP}$ and 20 LP flows, and (c) $20 \mathrm{HP}$ and $20 \mathrm{LP}$ flows. It is noted that in every network configuration the number of LP flows is greater than, or equal to, the number of HP flows which is consistent with the kind of network scenarios which will benefit from our MAC scheme.

\subsection{Throughput performance for network scenarios I and II}

We first evaluate the throughput performance of our scheme in comparison to the IEEE 802.11e MAC protocol. The total throughput of a traffic flow is the sum of its throughput in the CP and CFP. We realize that the duration of the CFP will have a significant impact on the throughput performance of the scheme, especially on the LP traffic flows. We know that our scheme divides the available CFP duration into equal time chunks amongst all the traffic flows (regardless of an HP or LP flow) present in the network at that point in time, i.e., every data flow in the network gets an equal TXOP. However, priority is given to the HP traffic flows by giving them the privilege of transmitting their data in their respective time slots before the LP traffic flows (i. e., HP data flows are polled before LP data flows by the
$\mathrm{HC})$. We expect to see an overall increase in LP traffic throughput in our scheme by virtue of the extra time allocation provisioned for such traffic during the CFP. Figures 7, 8, and 9 validate our expectation. Figures 7 and 8 reflect the HP and LP traffics throughput of our scheme (denoted in the graph as "new HP throughput" and "new LP throughput") in comparison with the HP and LP throughput of the standard 802.11e MAC protocol (denoted in the graph as "old HP throughput" and "old LP throughput") simulated under network scenario I with a CFP duration of 50 (time units) and $160 \mathrm{TU}$, respectively. It is noted that the superframe duration (or Beacon interval) is $200 \mathrm{TU}$ in both cases. Significant inferences include the following:

(i) In network scenario I, there is about an average $20 \%$ increase in LP traffic throughput in our scheme in comparison to 802.11e. This is expected, since our scheme provisions for extra time to transmit LP traffic in the CFP which 802.11e does not. This trend is evident in both cases where the CFP duration is set to 50 and $160 \mathrm{TU}$, respectively. However, it is to be noted that the throughput curve for LP users in both cases (CFP $=50$ and $160 \mathrm{TU})$ shows a downward trend as the number of flows in the network increases. This can be attributed partly due to an increase in collisions during the CP. The major impact is however due to the thin time slicing of dedicated time slots allocated to each LP traffic flow as the number of flows increase in the network. This is a necessary evil since we have to accommodate all traffic flows in the network and yet not increase the total time duration of CFP. Also note that we present the results of the worst case traffic scenario. In our simulation, nodes have data to send all the time (constant bit rate-CBR-traffic) leading to a claim on the time slot during CFP always. In reality, not all nodes will have data to send at all times, thereby potentially preventing such thin time slicing for the data carrying nodes during CFP.

Scenario-1Average Throughput(bits/sec) vs number of users for CFP $=160 \mathrm{TU}$

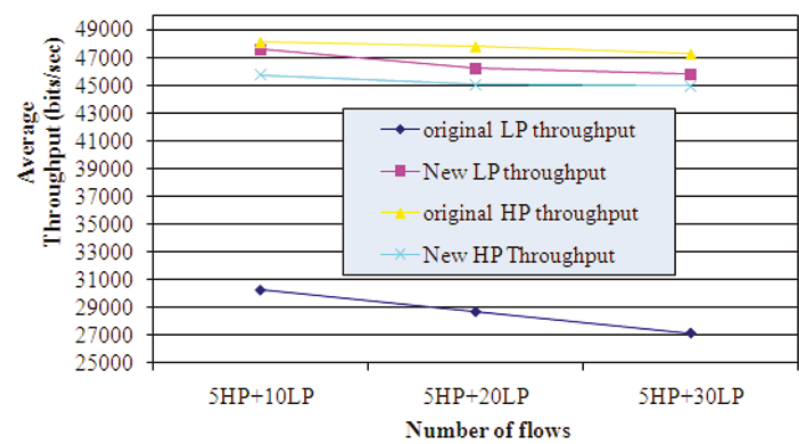

Figure 7 Average throughput of each traffic type for CFP of 50 TU. 


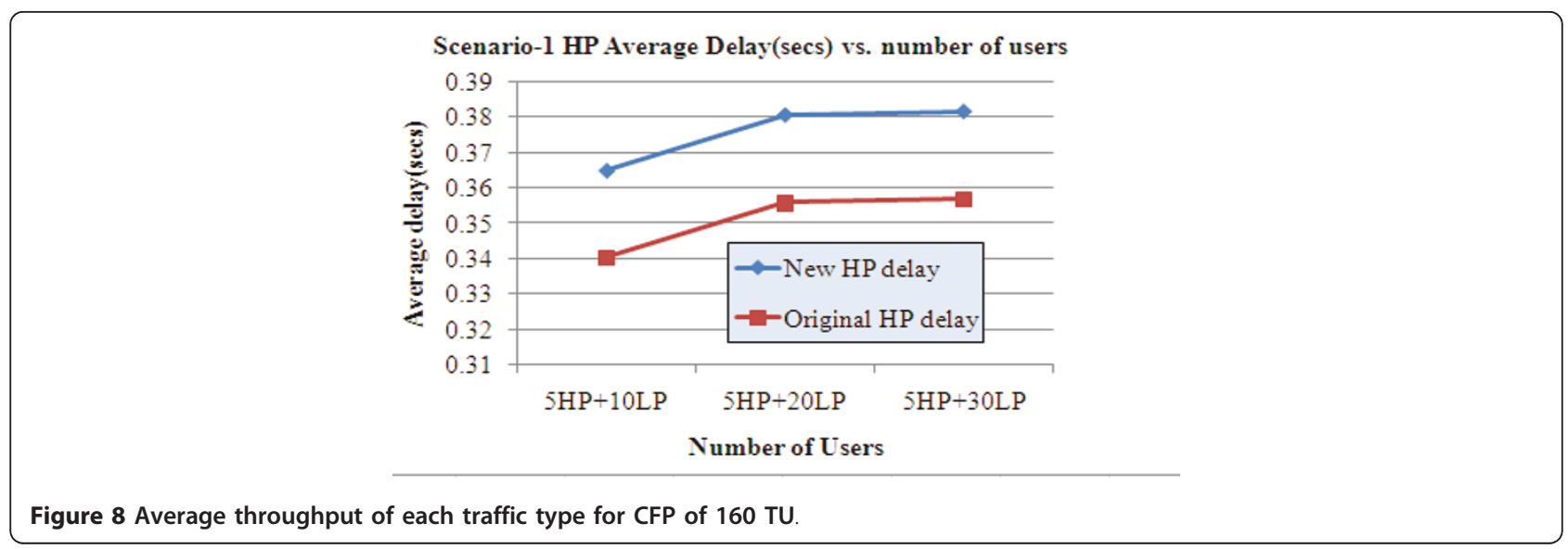

Figure 9 denotes the HP and LP throughput for network scenario II. This shows the same trend in throughput increase for LP traffic as in Figures 7 and 8 for the same reasons as explained in the previous paragraph. It is to be noted that with an overwhelming increase in HP traffic, LP throughput experiences a drastic decrease in standard 802.11e but not so in our scheme as a part of the CFP is still reserved for LP traffic transmission.

(ii) An increased duration of CFP in our scheme does lead to an increased throughput for both HP and LP traffic. If we increase the CFP duration to $160 \mathrm{TU}$, the throughput of our scheme increases about $50 \%$ more than the standard IEEE 802.11e scheme as depicted in Figure 8 . When the CFP duration is increased to 160 $\mathrm{TU}$ (superframe duration $=200 \mathrm{TU}$ ), there is a severe drop in LP throughput in the standard 802.11e due to the drastic increase in collisions during the $\mathrm{CP}$ which now comprises of a small fraction of time of the total superframe duration. This proves the relevance of CFP duration on the network performance. However, a longer CFP duration also increases the delay which can be detrimental, especially for HP traffic (Figures 10 and 11).

(iii) There is a $3 \%$ reduction in HP traffic throughput in our scheme in comparison to 802.11e. This is due to the thinner time slicing for each HP traffic flow during the CFP in our scheme to accommodate the extra LP flows within the stipulated CFP duration (for example 50 and $160 \mathrm{TU}$ in our simulations). It is noted that the increasing number of LP traffic flows also does not significantly affect the average HP throughput, since HP users contend for the channel more aggressively (better EDCA parameter set than LP) than the LP users and thus almost always gain access to the channel over LP users. The minimal decrease in throughput of HP traffic with an increase in the number of LP traffic flows (5 HP +10 LP flows configuration versus 5 HP + 30 LP flows network configuration) in Figures 7 and 8 is attributed to the smaller time segment devoted to each HP traffic flow during the CFP as the number of traffic flows in the system increases. In Figure 9, the drop in HP traffic throughput with an increase in the total number of

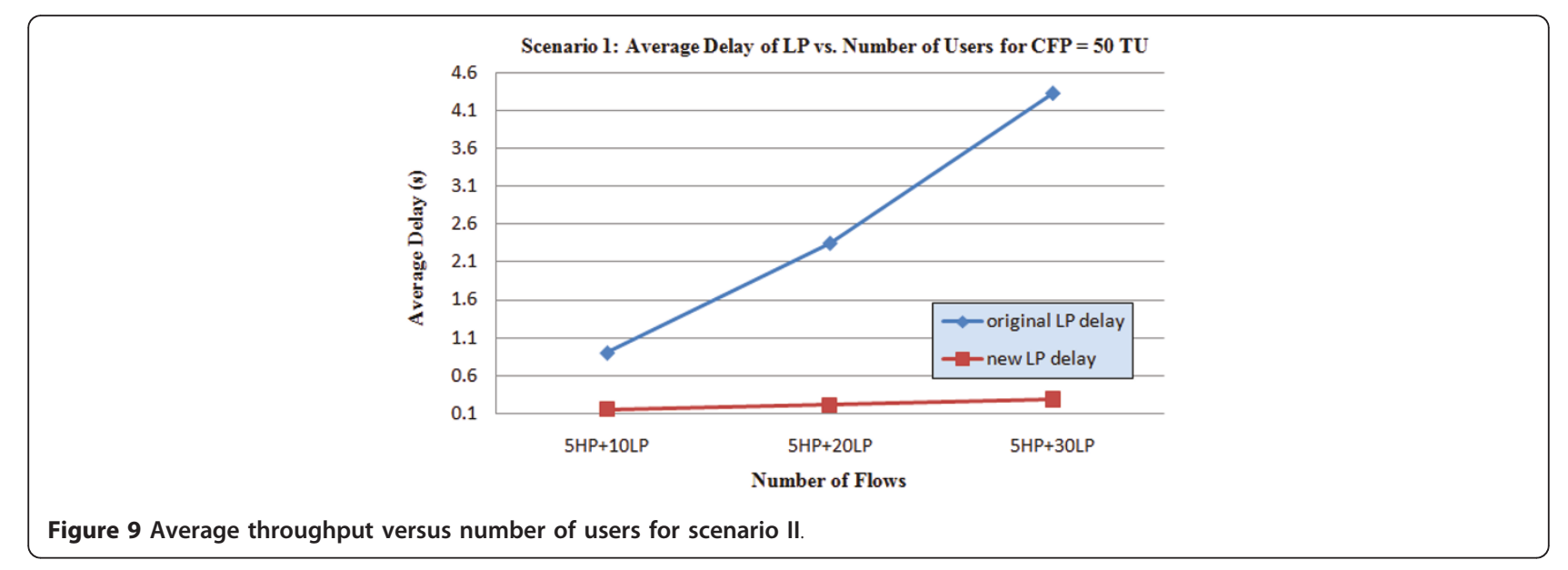




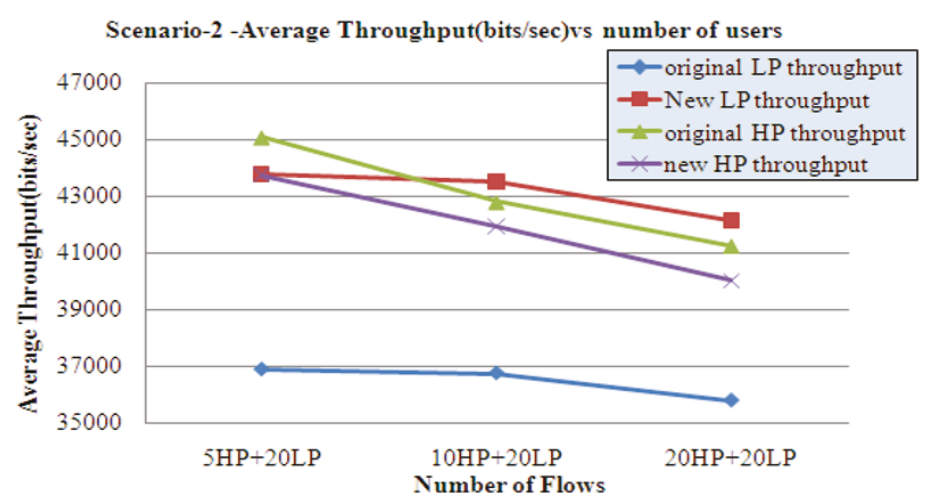

Figure 10 Average HP delay comparison between 802.11e and our scheme.

traffic flows in the network is attributed to the more aggressive channel contention that occurs between the increasing number of HP flows in the system, thereby leading to higher collisions and hence lesser overall HP throughput.

\subsection{Delay performance for network scenarios I and II}

Figures 10,1112, and 13 show the delay performance of HP and LP traffics, respectively, in our scheme (marked as "new delay" on the graph) in comparison to IEEE 802.11e (marked as "old delay" on the graph) for network scenarios I (Figures 10 and 12) and II (Figures 11 and 13). Our proposed scheme leads to about a $6 \%$ increase in delay for HP traffic, as expected, since the CFP phase is now utilized to serve the LP traffic as well in addition to the HP traffic, thereby leading to a longer wait time for the system to revert back to the $\mathrm{CP}$ where HP traffic can start their transmission again [20,21]. Specifically speaking, in our simulation setup (given our data rate of 2 Mbps and CBR packet arrival), we had set the delay bound of HP traffic to a reasonable $0.5 \mathrm{~s}$ for both traffic scenarios I and II. Simulation results depicted in Figures 10 and 11 validate that the HP delay stays within that bound. In general, any application that can tolerate a $7 \%$ increase in delay over a 2 Mbps data rate channel will not be adversely affected by our proposed scheme.

Moreover since majority of the flows are LP, this deterioration is compensated by the almost $20 \%$ increase in throughput of the LP traffic. Figures 12 and 13 demonstrate the fact that the delay of LP traffic decreases considerably in the proposed scheme when compared to the standard IEEE 802.11e.

In Figure 11, as the number of HP flows increase, the HP delay increases both in the standard 802.11e protocol and our scheme as well. This is due to the increased number of collisions during the CP as HP flows in the network increases thereby leading to longer wait time for data delivery. In network scenario II (Figure 11), the increase in HP delay over standard 802.11e is approximately less than $7 \%$. Once again, in scenarios where LP traffic predominates, this brunt in HP delay performance is acceptable, especially if we consider the havoc that can be wreaked in the network if selfish LP users start

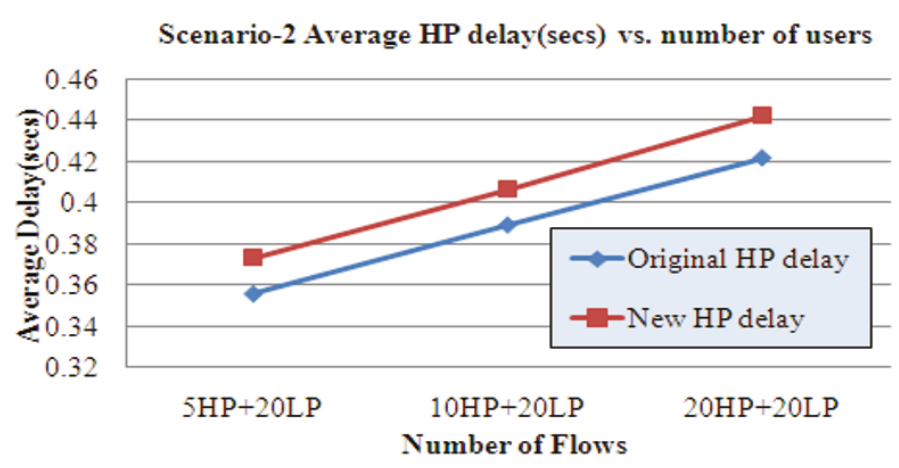

Figure 11 Average HP delay (s) versus number of users. 


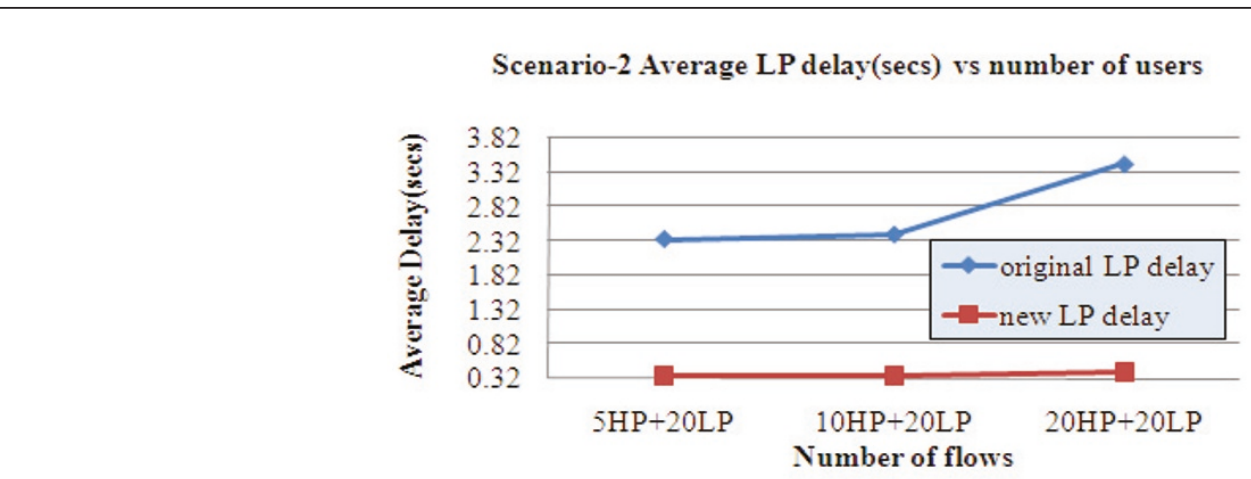

Figure 12 Average LP delay comparison between 802.11e and our scheme.

classifying their traffic as HP and thereby wreck the whole notion of QoS [8] in absence of a fair MAC scheme like ours.

\section{Conclusions}

This article focuses on "protecting" the "underdog" or non-real time data traffic in the face of the growing multimedia traffic that treads the wires in recent times. It provides analytical expressions to model the throughput and delay of a hybrid MAC scheme akin to IEEE 802.11e followed by MATLAB simulation results which highlight the drawback of protocols that are biased toward protecting and guaranteeing QoS for delay intolerant HP traffic thereby starving the delay tolerant nonreal time flows. In addition, this article proposes a MAC scheme which provides performance (throughput) guarantees to non-real time traffic in face of real-time traffic such that they are not bandwidth starved. However, the new MAC protocol geared toward protecting the "underdog" traffic also aims to preserve the QoS requirements of delay-intolerant, $\mathrm{HP}$. The performance of the new MAC scheme is compared against the standard IEEE 802.11e scheme using the QualNet simulation platform. The results prove that the proposed MAC scheme indeed boosts the throughput and delay performance of non-real time traffic (by as high as 50\%) with a minimal dent in throughput (about 3\%) and delay (about $6 \%$ ) of real-time traffic though staying within the acceptable service range of such traffic. The QualNet simulation results show that the performance improvement of our proposed method is particularly significant when the traffic mix comprises of mainly delay tolerant traffic. Conventionally, using schemes like IEEE 802.11e, LP traffic would have been sabotaged by a small population of HP traffic which would have conventionally squeezed the majority of network resources to ensure its high performance. Our protocol alleviates this particular problem and proves that a fairer scheme is indeed

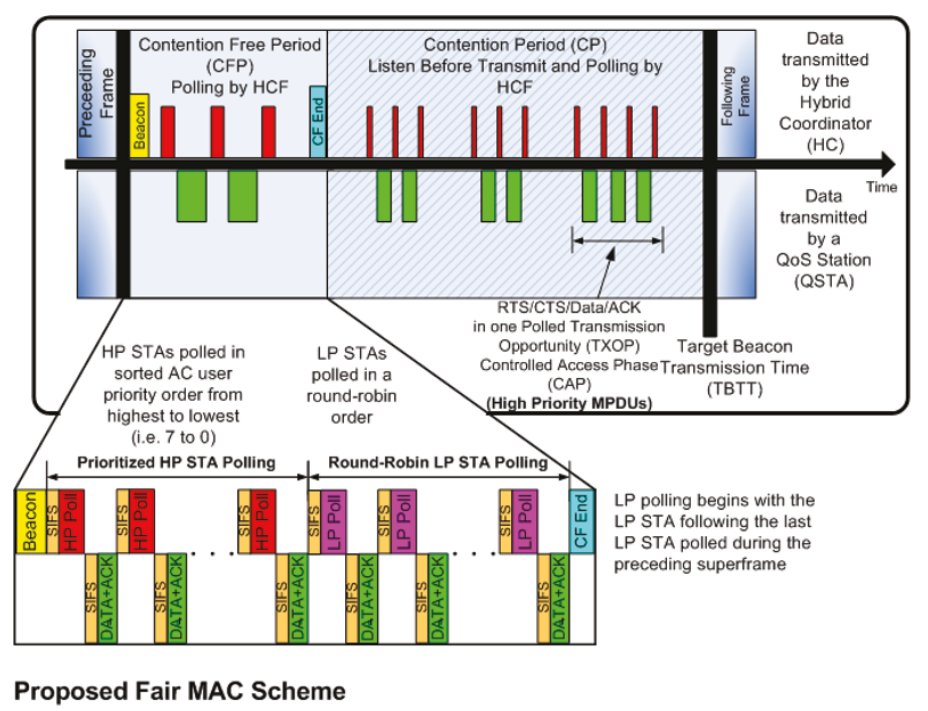

Figure 13 Delay performance of LP traffic. 
possible and complete sabotage of non-real time traffic is not required to meet the demands of high priority traffic.

\section{Acknowledgements}

This study is based upon work supported by the National Science

Foundation under Grant No. 0737048.

\section{Author details}

${ }^{1}$ Electrical and Computer Engineering, San Diego State University, 5500 Campanile Drive, San Diego, CA 92182, USA ${ }^{2}$ Computational Science Research Center, San Diego State University, 5500 Campanile Drive, San Diego, CA 92182, USA

\section{Competing interests}

The authors declare that they have no competing interests.

Received: 2 March 2011 Accepted: 12 October 2011

Published: 12 October 2011

\section{References}

1. itbusiness.ca-business advantage through Technology retrieved at http:// www.itbusiness.ca/it/client/en/home/News.asp?id=48078\&PageMem=3

2. pedistrict-news, retrieved at http://www.pcdistrict.com/internet-traffic-is-stilldominated-by-p2p-file-sharing-review1510-16.html

3. Medium Access Control (MAC) Quality of Service (QoS) Enhancements. IEEE 802.11e Working Group, New York, P802.11e/D13.0, (January 2005)

4. IEEE Standard for Information Technology-Part 11: Wireless LAN (MAC) and (PHY) Specifications. IEEE Std 802.11, 6/12/2007

5. O Ni, T Turletti, OOS support for IEEE 802.11 Wireless LAN (Nova Science Publishers, 2004)

6. A Lindgren, A Almquist, O Schelén, Quality of service schemes for IEEE 802.11 wireless LANs-an evaluation. J Mobile Netw Appl. 8(3), 223-235 (2003). doi:10.1023/A:1023389530496

7. P Garg, R Doshi, R Greene, M Baker, M Malek, X Cheng, Using IEEE 802.11e MAC for QOS over wireless, in Proceedings of IEEE Performance, Computing, and Communications Conference (April 2003)

8. P Nuggehalli, M Sarkar, RR Rao, QoS and selfish users: a MAC layer perspective, in Proc of IEEE GLOBECOM, Boston, MA (November 2007)

9. D Obeid, S Sheeshia, Modeling of a polling-based access scheme for 802.11, in Proceedings of the 32nd IEEE Conference on Local Computer Networks (2007)

10. M Sarkar, M Ray, P Nuggehalli, Evaluating a QoS-supportive MAC layer protocol for WLANs, in Proceedings of IEEE MILCOM 2008, San Diego, CA (2008)

11. BA Venkatakrishnan, S Selvakennedy, An enhanced HCF for IEEE 802.11e wireless networks, in Proceedings of the 7th ACM International Symposium on Modeling, Analysis and Simulation of Wireless and Mobile Systems, Venice, Italy (2004)

12. R Khalaf, I Rubin, throughput and delay analysis in single hop and multihop IEEE 802.11 networks, in Proc of 3rd International Conference on Broadband Communications, Networks and Systems, 2006 (BROADNETS 2006), San Jose, CA, 1-9 (2006)

13. HL Truong, G Vannuccini, The IEEE 802.11e MAC for Quality of Service in Wireless LANs, (IBM Research, Zurich Research Lab, Switzerland, 2003)

14. P Ansel, Q Ni, T Turletti, P Planete, FHCF, a fair scheduling scheme for 802.11e WLAN. INRIA Research Report 4883 (2003)

15. S Mangold, S Choi, G Hiertz, O Klein, B Walke, Analysis of IEEE 802.11 e for QoS support in wireless LANs. IEEE Wirel Commun Mag. 10(6), 40-50 (2003). doi:10.1109/MWC.2003.1265851

16. A Grilo, M Macedo, N Nunes, A scheduling algorithm for QoS support in IEEE 802.11e networks. IEEE Wirel Commun Mag. 10(3), 36-43 (2003). doi:10.1109/MWC.2003.1209594

17. D Gao, J Chai, L Zhang, Physical rate based admission control for HCCA in IEEE 802.11e WLANs, in Proceedings of the 19th international Conference on Advanced Information Networking and Applications (AINA), Taipei, Taiwan, 479-483 (March 2005)

18. QualNet 4.5", http://www.scalable-networks.com
19. G Bianchi, Performance analysis of the IEEE 802.11 distributed coordination function. IEEE J Sel Areas Commun. 18(3), 535-547 (2000). doi:10.1109/ 49.840210

20. R Al-Sayyed, C Pattinson, T Dacre, VolP and database traffic co-existence over IEEE 802.11b WLAN with redundancy, in Proceedings of the International Conference on Computer, Information and Systems Science and Engineering, Barcelona, Spain, 25-27 (April 2007)

21. DP Agrawal, Q Zeng, Introduction to Wireless and Mobile System, (Thomson, Brooks/Cole, 2003) ISBN: 0534-40851-6

doi:10.1186/1687-1499-2011-131

Cite this article as: Sarkar and Paolini: A QoS guaranteeing MAC layer protocol for the "underdog" traffic. EURASIP Journal on Wireless

Communications and Networking 2011 2011:131.

\section{Submit your manuscript to a SpringerOpen ${ }^{\mathcal{O}}$ journal and benefit from:}

- Convenient online submission

- Rigorous peer review

- Immediate publication on acceptance

- Open access: articles freely available online

- High visibility within the field

- Retaining the copyright to your article

Submit your next manuscript at $\gg$ springeropen.com 